\title{
Calcificación pericárdica: ¡no se precipite! La importancia de la valoración hemodinámica invasiva
}

\author{
Pericardial calcification: Don't rush! The importance of invasive \\ hemodynamic assessment
}

\author{
Juan Ruiz-García ${ }^{a, b, c, *}$, Irene Canal-Fontcubertad ${ }^{d}$ Atenea Rodríguez-Salgado ${ }^{a}$, \\ David Sánchez-Roncero ${ }^{a}$, Paloma Ávila-Barahona ${ }^{a}$ y Eduardo Alegría-Barrero ${ }^{a}, b, c$ \\ a Unidad de Hemodinámica, Servicio de Cardiología, Hospital Universitario de Torrejón, Torrejón de Ardoz, Madrid, España \\ b Unidad de Cardiología, Hospital Ruber Internacional, Madrid, España

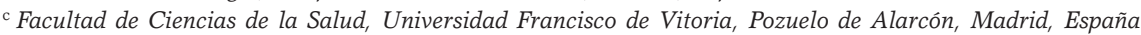 \\ d Servicio de Oftalmología, Hospital Universitario de Torrejón, Torrejón de Ardoz, Madrid, España
}

\section{Sr. Editor:}

La pericarditis constrictiva $(\mathrm{PC})$ es una causa de insuficiencia cardiaca diastólica secundaria a la presencia de un pericardio no distensible. La sospecha diagnóstica resulta fundamental, pues si bien es una afección potencialmente curable, se asocia a una elevada morbimortalidad si no se trata ${ }^{1}$. Su diagnóstico diferencial con la miocardiopatía restrictiva puede suponer un auténtico reto ${ }^{2}$. Se presenta un caso en el que el estudio hemodinámico invasivo mediante cateterismo cardiaco demostró su vigencia e importancia diagnóstica en la toma de decisiones terapéuticas.

Se describe el caso de una mujer de 59 años, hipertensa y exfumadora, con antecedentes de linfoma de Hodgkin a los 18 años - por el que recibió telecobaltoterapia y quimioterapia- y de carcinoma localizado de mama izquierda tratado con mastectomía a los 52 años. Reingresó por segunda vez en 2 meses debido a un cuadro grave de insuficiencia cardiaca derecha con presencia de signo de Kussmaul (vídeo 1 del material adicional). Se realizó una ecocardiografía transtorácica que, debido a la pésima ventana acústica, solo mostró un engrosamiento pericárdico y una función sistólica biventricular preservada sin valvulopatías significativas.
Ante la existencia de una calcificación grave pericárdica (figura 1), su clínica y su historia de radiación torácica, se solicitó un cateterismo cardiaco para confirmar la sospecha de PC posradioterapia e indicar una pericardiectomía quirúrgica.

El estudio hemodinámico determinó un índice cardiaco reducido $\left(1,82 \mathrm{l} / \mathrm{min} / \mathrm{m}^{2}\right)$ y un importante aumento de presiones en las cavidades cardiacas derechas, y mostró otros signos clásicos de pericarditis constrictiva ${ }^{1-3}$, como los senos $\mathrm{x}$ e y marcadamente profundos en la aurícula derecha (figura 2A) y la morfología dipplateau en la curva de presión del ventrículo derecho (figura 2B). El registro simultáneo de las presiones de ambos ventrículos permitió visualizar también la igualación de sus presiones telediastólicas (figura 2C), aunque descartó el esperado aumento de interdependencia ventricular, con la existencia de cambios en paralelo en ambas presiones con los movimientos respiratorios (figura 2D). La paciente presentaba además una hipertensión pulmonar moderada poscapilar (figura $2 \mathrm{E}$ y $2 \mathrm{~F}$ ). No se encontraron lesiones coronarias significativas, aunque las ramas más distales de la arteria coronaria derecha se encontraban «fijadas» a nivel de la calcificación pericárdica inferior (figura 1 y vídeo 2 del material adicional), un hallazgo considerado específico de $\mathrm{PC}^{1}$.

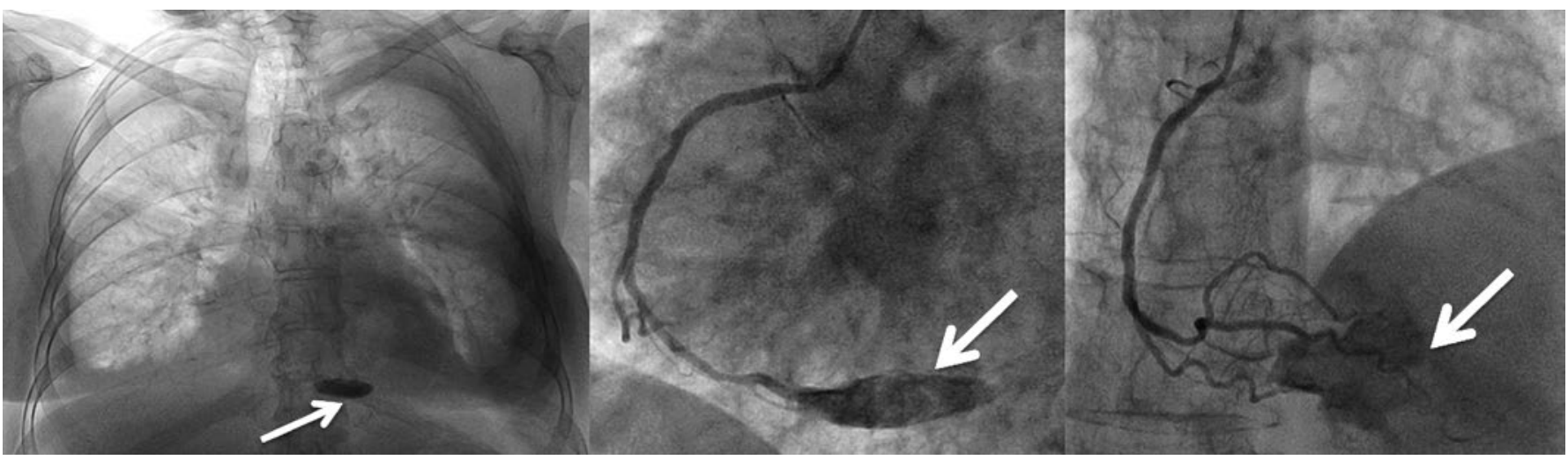

Figura 1. Calcificación pericárdica inferior (flechas).

\footnotetext{
* Autor para correspondencia: Servicio de Cardiología, Hospital Universitario de Torrejón, Mateo Inurria s/n, 28850 Torrejón de Ardoz, Madrid, España. Correo electrónico: j.ruizgarcia@hotmail.com (J. Ruiz-García). 


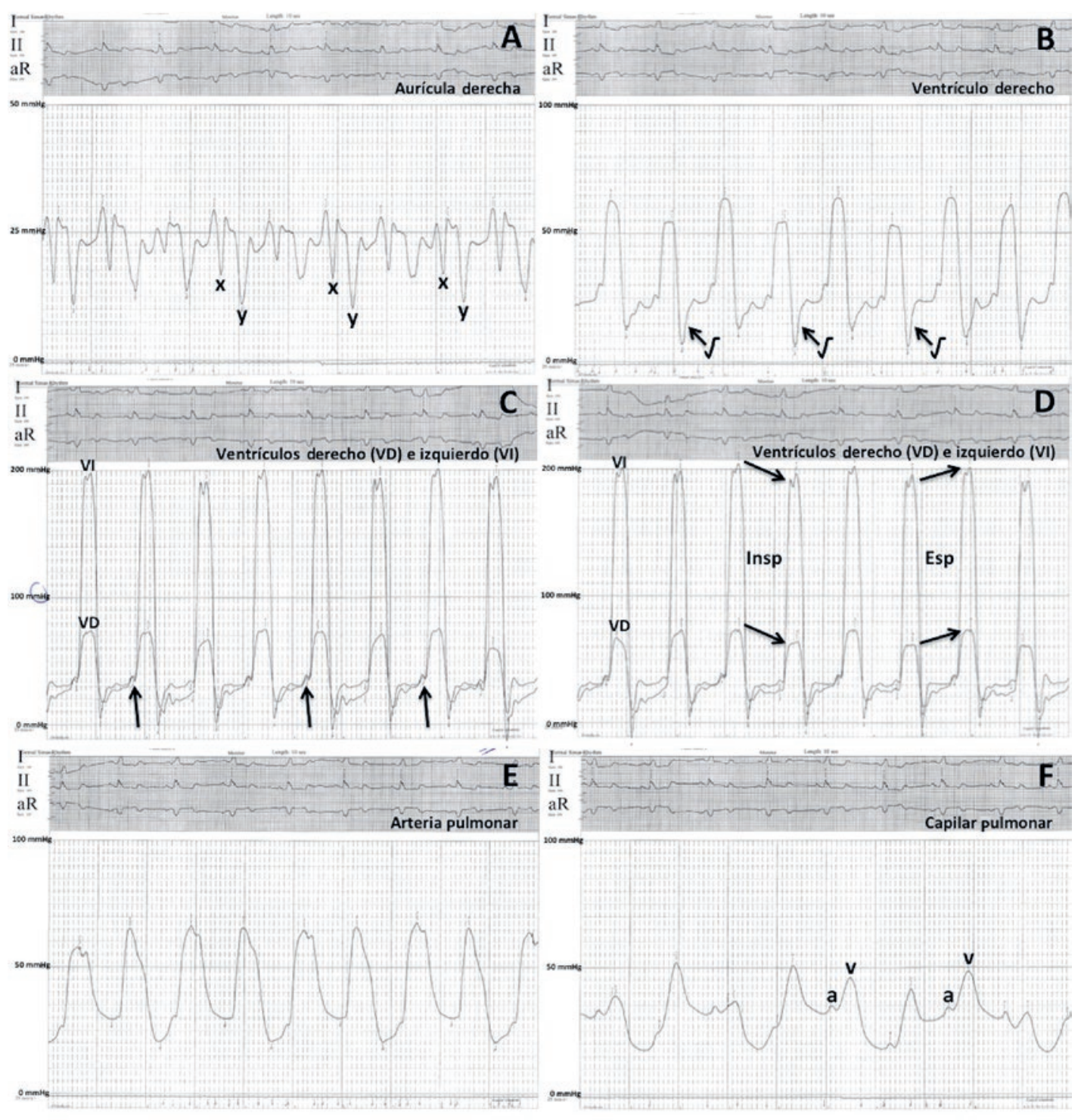

Figura 2. Registro de presiones. A: aurícula derecha (media $22 \mathrm{mmHg}$ ) con senos x e y profundos; B: ventrículo derecho (61/10-27 mmHg) con signo de raíz cuadrada (flecha); C y D: ventrículos derecho e izquierdo con igualación de ambas presiones telediastólicas (flechas), pero con descenso en paralelo (concordancia ventricular) de las curvas de presión durante la inspiración (Insp) y aumento en paralelo durante la espiración (Esp); E: arteria pulmonar $(65 / 25(42) \mathrm{mmHg})$; y F: capilar pulmonar (31 $\mathrm{mmHg}$ ) con onda v prominente.

Debe considerarse el diagnóstico de PC ante la presencia de síntomas de insuficiencia cardiaca fundamentalmente derecha. Los hallazgos clínicos y las pruebas diagnósticas no invasivas permiten diagnosticarla en el $70 \%$ de los $\operatorname{casos}^{1}$. Sin embargo, el resto de los pacientes podrían necesitar un cateterismo cardiaco para la correcta diferenciación entre PC y miocardiopatía restrictiva, muy especialmente aquellos con antecedente de radioterapia o de cirugía cardiaca en los que a menudo coexisten las afectaciones pericárdica y miocárdica ${ }^{2,3}$.

Los criterios clásicos de PC (p. ej., llenado ventricular rápido precoz o ecualización de las presiones telediastólicas de las 4 cámaras cardiacas, ausencia de hipertensión pulmonar, etc.) tienen unas bajas sensibilidad y especificidad para realizar el diagnóstico diferencial con la miocardiopatía restrictiva ${ }^{1-3}$. En la actualidad se considera que las variaciones respiratorias dinámicas de las presiones intracardiacas tienen una gran precisión ${ }^{1,2}$, con una sensibilidad del $97 \%$ y un valor predictivo positivo del $100 \%$, para identificar pacientes con $\mathrm{PC}^{3}$. En la $\mathrm{PC}$ hay una disociación entre las presiones intratorácicas e intracardiacas, con una reducción en la presión intratorácica que no se transmite a las cavidades cardiacas durante la inspiración, lo que conduce a una reducción en el llenado del ventrículo izquierdo. En presencia de un pericardio no distensible, con un volumen intrapericárdico relativamente fijo, la reducción del llenado del ventrículo izquierdo aumenta simultáneamente el llenado del ventrículo derecho. Con la espiración ocurre lo contrario. Este aumento de la interdependencia ventricular se traduce directamente en una alteración de las presiones sistólicas ventriculares. En otros escenarios clínicos hay un aumento y un descenso de ambas presiones en paralelo con el ciclo respiratorio (concordancia ventricular), pero en el caso de la PC esas variaciones son discordantes ${ }^{1,2}$, lo cual es un marcador altamente sensible y específico ${ }^{3}$ que no se logró encontrar en nuestra paciente.

Si bien el ecocardiograma Doppler puede valorar estos cambios con la fase respiratoria mediante el análisis del flujo transmitral y los movimientos del tabique ${ }^{1}$, es habitual que los pacientes que han sido sometidos a cirugía cardiaca o a radioterapia presenten 
ventanas acústicas que compliquen o impidan su valoración. Como se ha comprobado, la detección de un engrosamiento o de una calcificación pericárdica en las diferentes pruebas de imagen tampoco refleja necesariamente una fisiopatología puramente constrictiva ${ }^{3}$.

Nuestro estudio hemodinámico evidencia una probable forma mixta de constricción y restricción miocárdica secundaria a la radiación torácica sufrida por la paciente en su juventud. Con los datos presentados en este trabajo, creemos que el caso refuerza la necesidad de realizar una evaluación hemodinámica muy precisa de estos pacientes antes de indicar cualquier intervención quirúrgica ${ }^{2}$, más aún cuando la mortalidad perioperatoria de la pericardiectomía en ellos puede superar el $20 \%{ }^{1}$. Por ello, se optó inicialmente por una actitud conservadora, en espera de la evolución clínica tras una lenta y adecuada optimización del tratamiento médico.

\section{MATERIAL ADICIONAL}

Se puede consultar material adicional a este artículo en su versión electrónica disponible en https://doi.org/10. 24875/RECIC.M19000022.

\section{BIBLIOGRAFÍA}

1. Miranda WR, Oh JK. Constrictive Pericarditis: A Practical Clinical Approach. Prog Cardiovasc Dis. 2017;59:369-379.

2. Geske JB, Anavekar NS, Nishimura RA, Oh JK, Gersh BJ. Differentiation of Constriction and Restriction: Complex Cardiovascular Hemodynamics. J Am Coll Cardiol. 2016;68:2329-2347.

3. Talreja DR, Nishimura RA, Oh JK, Holmes DR. Constrictive pericarditis in the modern era: novel criteria for diagnosis in the cardiac catheterization laboratory. J Am Coll Cardiol. 2008;51:315-319.

\title{
Seudoaneurisma de arteria pulmonar como complicación de catéter de Swan-Ganz
}

\section{Pulmonary artery pseudoaneurysm as a Swan-Ganz catheter complication}

\author{
Laura Gallego López a,*, Aurora Díaz Valdecantos ${ }^{\mathrm{b}}$, César Carrascosa Rosilloc \\ y Nuria Miranda Balbuena ${ }^{\mathrm{d}}$ \\ a Servicio de Medicina Interna, Hospital Universitario Virgen Macarena, Sevilla, España \\ b Servicio de Cardiología, Hospital Universitario Virgen Macarena, Sevilla, España \\ c Servicio de Hemodinámica, Hospital Universitario Virgen Macarena, Sevilla, España \\ d Servicio de Cirugía Cardiovascular, Hospital Universitario Virgen Macarena, Sevilla, España
}

\section{Sr. Editor:}

Se presenta el caso de una mujer de 65 años sin alergias conocidas a medicamentos y con antecedentes personales cardiológicos de fibrilación auricular, en tratamiento anticoagulante con warfarina, y con polivalvulopatía reumática con doble lesión mitral (estenosis mitral grave e insuficiencia mitral moderada-grave) e insuficiencia tricuspídea grave con datos indirectos de hipertensión pulmonar, que consultó por un aumento progresivo de su disnea habitual hasta hacerse de mínimos esfuerzos y con importantes limitaciones en su vida diaria.

Fue valorada en el servicio de cirugía cardiovascular y se procedió a la sustitución de la válvula mitral por una prótesis mecánica y anuloplastia tricuspídea el 17 de abril de 2018, sin incidencias intraoperatorias ni complicaciones en el posoperatorio inmediato en la unidad de cuidados intensivos y posteriormente en planta, de donde fue dada de alta hospitalaria.
El 31 de mayo de 2018 la paciente acudió al servicio de urgencias por un incremento de su disnea hasta hacerse de moderados esfuerzos, con cierto aumento del perímetro abdominal y edemas en ambos miembros inferiores. La analítica destacó un propéptido natriurético elevado. La radiografía de tórax mostró signos congestivos y llamaba la atención una imagen nodular en la base pulmonar derecha, sugestiva de tumor evanescente, no observada en estudios radiológicos previos. Tras el tratamiento depletivo se realizó otra radiografía de tórax de control, en la que persistía una imagen redondeada y bien delimitada, por lo que se solicitó una tomografía computarizada de tórax que mostró un seudoaneurisma de arteria pulmonar parcialmente trombosado.

Se comentó el caso con el servicio de hemodinámica y se llevó a cabo la embolización del seudoaneurisma el 20 de junio de 2018 . Mediante acceso femoral derecho de $5 \mathrm{Fr}$ se procedió a acceder con catéter diagnóstico 5F JR 4 hasta la arteria pulmonar derecha, y una vez allí se intercambió sobre una guía 0035 con catéter MP

\footnotetext{
* Autor para correspondencia: Servicio de Medicina Interna, Hospital Universitario Virgen Macarena, Roelas 1 Bajo, 41002 Sevilla, España. Correo electrónico: laura.gallego.lopez9@gmail.com (L. Gallego López). 